\title{
Measurement of acetates in air using differential ion mobility spectrometer
}

\author{
Andrzej Szczurek ${ }^{1}$, Monika Maciejewska ${ }^{1, *}$, Żaneta Zajiczek ${ }^{1}$ and Mirosław Maziejuk ${ }^{2}$ \\ ${ }^{1}$ Wroclaw University of Science and Technology, Faculty of Environmental Engineering, Wybrzeże \\ Wyspiańskiego 27, 50-370 Wrocław, Poland \\ ${ }^{2}$ Military Institute of Chemistry and Radiometry, al. Chruściela 105, 00-910Warsaw, Poland
}

\begin{abstract}
Volatile organic compounds are one of the most important group of air pollutants. Potential health and environmental problems resulting from their emission prompted the requirement for monitoring these species. It motivates development of new measurement techniques which are fast, cost effective, reliable and field deployable. One of novel approaches is ion mobility spectrometry. It dwells on ion separation in electric field, based on differences in ion mobility. Many variants of this method are developed. In this wok, differential ion mobility spectrometry (DMS) was considered in respect of acetate measurements in air. It was demonstrated that DMS offers linear response to methyl, ethyl, propyl and butyl acetate in concentration range from $0.3 \mathrm{ppm}$ to $7 \mathrm{ppm}$. Positive ions spectrum has to be utilised for this purpose. We showed that fragments of DMS spectrum which secure linearity are compound-specific. The obtained results are promising from the application point of view.
\end{abstract}

\section{Introduction}

Volatile organic compounds (VOCs) are believed to have short-term and long-term adverse effects on environment and human health. Therefore they are classified as a one of the most important group of air pollutants. Although natural (biological) emissions tend to be larger anthropogenic sources are a greater concern in many environments. VOCs are emitted to the atmosphere from a number of outdoor and indoor sources [1,2]. Typical outdoor sources are industrial processes (especially those involving solvents e.g. printing, spray painting, coil coating, wood treatment etc.), vehicle emissions, evaporative loss from petrol storage, and evaporation from landfill sites. The key indoor sources include paints, cleaning supplies, furnishings, glues, permanent markers and printing equipment. They are particularly important when ventilation is limited.

VOCs are important environmental contaminants because of their mobile, persistent and toxic properties. Some of them are harmful to human health, with several classified as carcinogenic (e.g. benzene) and toxic (e.g. methyl alcohol). Prolonged human exposure to VOCs can lead to throat and eyes irritation, respiratory problems, headaches and liver, kidneys, or central nervous system damage. These substances can also cause serious

\footnotetext{
*Corresponding author: author@email.org
} 
environmental damage [3]. They play important roles in stratospheric ozone depletion, contribute to local or transboundary formation of photochemical oxidants in the boundary layer of the troposphere, leading to environmental degradation, enhancing the global greenhouse effect. VOCs have a potentially negative impact on industrial safety. A lot of them are fire hazards or can form an explosive mixture in air.

Potential health and environmental problems resulting from the emission and use of VOCs has prompted requirement to monitor these pollutants, particularly in relation to compliance with limit values, determination of the emission control effectiveness, risk assessment or assessing trends in time or space. A requirement to monitor implies the availability of well establish analytical methods and techniques. Today, there are a number of instruments capable of identifying and quantitating specific chemical species. If qualitative and quantitative analysis is required then infrared analyzers, Fourier-transform infrared spectrometers (FT-IR spectrometers) or gas chromatographs with appropriate detector (usually FID or tuned mass spectrometer MS or other mass-selective detector) can be employed $[4,5]$. These instruments offer very good accuracy, sensitivity, selectivity and repeatability. Unfortunately, they are expensive, time-consuming and require trained, experienced personnel. An alternative method is the electronic nose (e-nose) system that consists of an array of chemical gas sensors [6]. However, different VOCs may produce similar patterns, thus the discrimination capability of this approach is limited. The problems with targeted VOC measurements cause that determination of total VOCs is acceptable to comply with legislation. The two most widely used technologies to measure total VOCs are Flame Ionization Detection (FID) and Photo Ionization Detection (PID).

Large demand for sensitive and selective detection of VOCs in the gas phase for routine laboratory analysis, emission determination, industrial processes examinations, safety monitoring, surveillance and homeland security is so important, that novel methods and techniques are required. They should be objective, fast, cost effective, reliable and field deployable. It is worth mentioning that less accurate, but simpler, quicker and more convenient methods are entirely satisfactory and desirable for many applications.

One of novel approaches is based on a Ion Mobility Spectrometry (IMS). It stands for a set of laws, methods and instrumentation which may be used to characterize analytes based on the movement of ensembles of gaseous ions derived from these analytes in an electric field, through a supporting gas atmosphere [7]. This technology combines high sensitivity and relatively low cost of a single analysis with a fast response to tested gas. Unfortunately, drawbacks such as low resolving power (not very high selectivity ), high false positive rates, weaknesses in the robustness regarding a poor linear range, "memory effects" from contamination and interference from matrices reduce the applicability of the IMS system for detection of harmful chemicals. Recently, differential mobility spectrometry (DMS) has proven to be a valuable addition to mobility spectrometry (MS) analyses.

Differential mobility spectrometry (DMS) is a method of separating ions based on the difference between ion mobility in high and low electric fields in gases at or near atmospheric pressure. DMS separates ions based upon subtle differences in their chemical structures [7-10]. For the development of DMS, it is important to know what role in the mechanism of analytical signal generation play functional groups. In organic chemistry, functional group is defined as the defined group of atoms within molecule, arranged in a specific manner that gives the compound its characteristic properties. It is the centre of chemical reactivity. The same functional group will undergo the same or similar chemical reaction(s) regardless of the size of the molecule it is a part of. It is important to recognize the influence of various functional groups on differential mobility spectra $[11,12]$.

The aim of this paper is to demonstrate that DMS allows to distinguish organic compounds that contain various functional groups and determine their concentration. Our study was focused on acetates. These compounds have found broad application in textile 
industry. They are widely used as solvents, precursors of important chemicals, essential ingredients in many products and materials e.g. in coatings, inks and fragrances in food production. Acetates are popular because they have inoffensive, often sweet odors, they are inexpensive, and they usually have low toxicity. Various reasons cause that information about these compound is needed. Acetates examined in this work are described by the following general formula: $\mathrm{CH}_{3} \mathrm{COO}-\mathrm{R}$. In this study, we focused on the following alkyl groups $\mathrm{R}$, methyl $\left(\mathrm{CH}_{3}-\right)$, ethyl $\left(\mathrm{C}_{2} \mathrm{H}_{5}-\right)$, propyl $\left(\mathrm{C}_{3} \mathrm{H}_{7}-\right)$ and butyl $\left(\mathrm{C}_{4} \mathrm{H}_{9}-\right)$. The functional groups included in the molecules of acetates can be precursors of different ions which are responsible for differential mobility spectra. On this basis, there is possible to perform quantitative and qualitative analysis of acetates in air.

\section{Experimental part}

\subsection{Differential ion mobility spectrometer}

Differential ion mobility spectrometry (DMS) is one of approaches developed in the domain of IMS. It is founded on the observation that in strong electric fields (exceeding 12 $\mathrm{kV} / \mathrm{cm}$ ) ion mobility displays nonlinear increase with the field [8]. This property is applied for ions separation and characterization.

Essential issues in DMS development are: 1) ionization of gas sample 2) evoking oscilating movement of ions in high amplitude, high frequency, asymmetric electric field caused by the potential difference, called separation voltage; 3) ions drift compensation by applying constant component, called compensation voltage, which prevents their neutralization on chamber electrodes, 4) collection of ions which passed the drift region on collecting electrodes. Ions which have different mobility can be passed through the drift area at different compensation voltages.

DMS technique may be applied for the quantitative characterization of gas samples. If the residence time of sample molecules in reaction region is fixed, the peak intensity of product ions in the mobility spectrum is proportional to the concentration of sample molecules. However, the linear range of the relationship is limited by a number of factors. A very important one is spectrum variation upon the analyte concentration change. Upon sample concentration increase, there are formed proton bound dimers, trimers and even more complex ions in addition to protonated monomers.

In this work, we focussed on demonstrating that there are fragments of DMS spectrum where the instrument response is proportional to the concentration of selected acetates. Moreover, these fragments are compound-specific.

Measurements were performed with the DMS spectrometer, construction of the Military Institute of Chemistry and Radiometry, Poland. [13]. In the device, gas ionization is achieved by the $\beta$-emitter, nickel electrode covered with radioactive nickel ${ }^{63} \mathrm{Ni}$. The DMS spectrometer itself has planar geometry. The drift region is formed by two electrode-plates ( $5 \times 25 \mathrm{~mm}$ ) separated by a $0.5 \mathrm{~mm}$ gap. A high amplitude, high frequency, asymmetric voltage waveform, generated by HSV generator $(2 \mathrm{MHz})$ is applied to electrodes, crosswise to the direction of the carrier gas flow. The separation voltage amplitude is from 100 to $1600 \mathrm{~V}$ (peak to peak), which corresponds to from 6,5 to $104 \mathrm{Td}$. Additionally, constant weak compensation voltage is applied to electrodes. The compensation voltage is from -30 to $8 \mathrm{~V}$. At the end of the drift zone there are located two collecting electrodes $(5 \times 5 \mathrm{~mm})$, for collecting positive and negative ions respectively. Two types of ions are neutralized in parallel. The internal gas flow through the detector is $2.0 \mathrm{~L} / \mathrm{min}$. Chamber entrance temperature is $318 \mathrm{~K}$, chamber temperature is $323 \mathrm{~K}$. 


\subsection{Measurements}

Volatile organic compounds considered in this study were methyl, ethyl, propyl and butyl acetate. They were examined in the following concertation ranges: methyl acetate (from 0.21 to $6.15 \mathrm{ppm}$ ), ethyl acetate (from 0.25 to $6.08 \mathrm{ppm}$ ), propyl acetate (from 0.22 to 4.28 ppm) and butyl acetate (from 0.39 to $5.44 \mathrm{ppm}$ ).

Standard gas mixtures containing individual acetates were prepared using an evaporation method. The setup dedicated to gas mixtures preparation was a part of the measurement setup, shown in Fig. 1. In the setup, purified air was used as the carrier gas and the cleaning agent. Purified air was attained by blowing room air through a set of filters, with a pump.

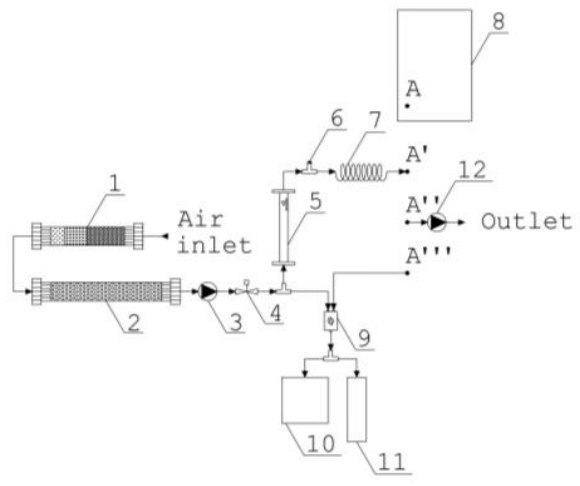

Fig. 1. Measurement set-up. It consists of the following elements: 1. filter (active carbon, molecular sieve, soda lime), 2. filter (silica gel), 3. membrane pump, 4. regulation valve, 5. rotameter, 6. Tconnector, 7. Teflon coil, 8- Tedlar bag (50 L), 9- two-way valve, 10-DMS spectrometer, 11-PID meter, 12- membrane pump.

For preparing standard gas mixture, the adequate amount of liquid analyte was injected, with the syringe, to the injection port mounted in Teflon tubing. The analyte evaporated while purified air passed through Teflon tubing and the coil. The resulting gas mixture was collected in the Tedlar bag (position A'). The purified air flow was adjusted by the valve and controlled by the rotameter. The concentration of analyte in gas mixture was estimated based on the amount of injected analyte, gas flow and its duration.

During measurement, gas mixture from Tedlar bag (position A') was continuously delivered to the spectrometer (constant rate $(0.5 \mathrm{l} / \mathrm{min})$ ) and photoionization detector (PID), in parallel. The time required to measure an individual gas sample with the spectrometer was $30 \mathrm{~s}$. In this period of time the separation voltage was changed (in steps equal $25 \mathrm{~V}$ ) and at each discrete value of separation voltage full range of compensation voltage was scanned (in steps equal $74 \mathrm{mV}$ ). The response, ionic current was measured on both collecting electrodes for each combination of separation and compensation voltage. Subsequent gas samples were measured with a time interval of $2 \mathrm{~min}$. On average, standard gas mixture was sampled 15 times, although in individual cases there were made from 6 to 20 repetitions. PID meter was employed as reference, to provide the true concertation of analyte in gas mixture. The device operated in a continuous manner and the measurement data was recorded with time resolution of $1 \mathrm{~s}$.

Prior to measurements and after measuring each standard gas mixture, Tedlar bag used for its preparation was cleaned by filling the bag with pure air and emptying it (position A'). The operation was repeated three times. Measuring instruments were cleaned by blowing purified air through them. This operation was performed prior, between and after measurements of standards gas mixtures. 


\section{Approach to measurement data analysis}

DMS spectrum consists of values ionic current recorded on the collecting electrode of DMS spectrometer in the predefined range of separation voltage (SV) and compensation voltage (CV). For data recording purposes these voltages are discretized, which results in a finite and countable set of combinations (SV, CV). The aim of measurement data analysis performed in this work was to evaluate all (SV, CV) combinations regarding linearity of the relationship between the concentration of analyte in gas sample and the ionic current on the collecting electrode. For this purpose, we applied univariate linear regression represented by the following formula

$$
c_{A}=a_{o}+a_{1} I_{(S V, C V)}+\varepsilon
$$

where $c_{A}$ is the concentration of particular analyte, $I_{(S V, C V)}$ is the ionic current recorded in response to analyte concentration $c_{A}$ at voltage combination (SV, CV), $a_{0}$ and $a_{1}$ are model coefficients and $\varepsilon$ is random error.

The quality of the linear fit between analyte concentration and ionic current was evaluated using coefficient of determination $\left(\mathrm{R}^{2}\right)$ and root mean square error of concentration assessment (RMSE). Both performance measures were determined for the complete set of measurements data applied for model parameterization.

\section{Results and discussion}

The results presented in this section are based on positive ions DMS spectra. In case of negative ions, there was not observed satisfactory degree of linearity between analyte concentration and the instrument response. Promising results were achieved exclusively for positive ions.

In Fig. 2 there are presented DMS spectra of purified air (Fig. 2a) and standard gas mixture, which contained methyl acetate at the concentration of $1.33 \mathrm{ppm}$ (Fig. 2b). Based on the difference between plots, it may be observed that DMS spectrometer responded to methyl acetate. As a result of positive reactant ions interaction with the analyte molecules, $\mathrm{M}$, at least the protonated molecules $\mathrm{MH}^{+}$, also called monomer ion were formed and recorded.
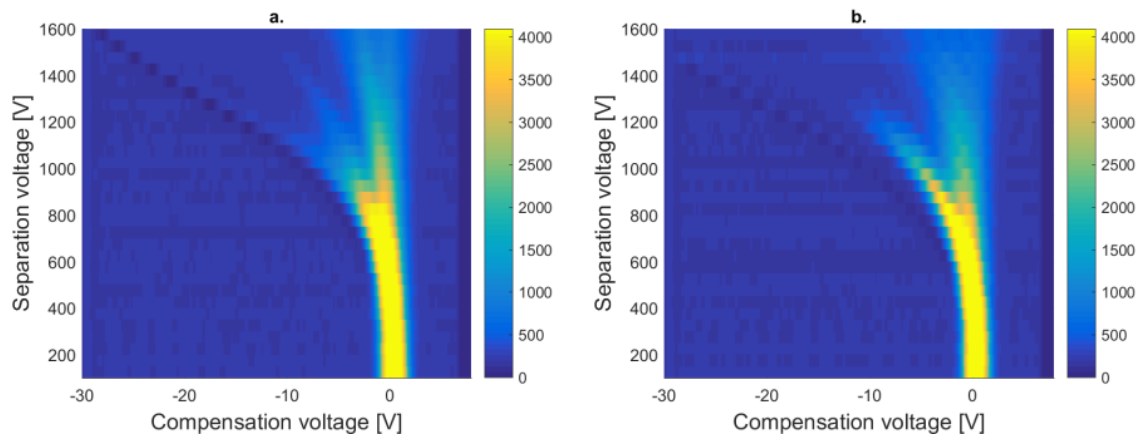

Fig. 2. DMS spectrum of positive ions recorded in response to a. purified air, b. gas mixture containing methyl acetate at the concentration of $1.33 \mathrm{ppm}$. 


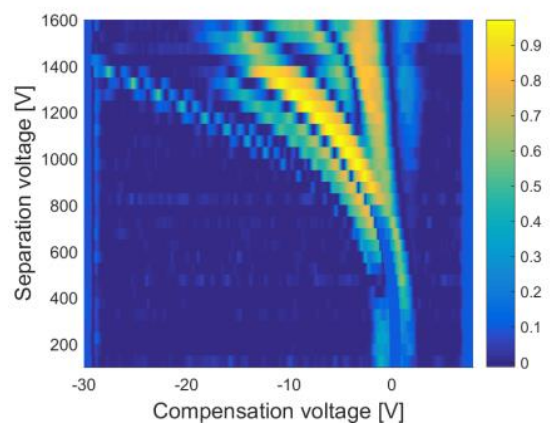

Fig. 3..Mapping of $\mathrm{R}^{2}$ coefficient in (SV,CV) domain, for ethyl acetate measured with DMS spectrometer, using positive ions.

In Fig. 3. there is shown mapping of $\mathrm{R}^{2}$ coefficient in $(\mathrm{SV}, \mathrm{CV})$ domain, for ethyl acetate. It is representative for the results obtained for other acetates. Based on Fig. 2b. there are zones in the spectrum, where the DMS response may be considered as linearly proportional to the concentration of analyte. They are indicated by high values of $\mathrm{R}^{2}$ coefficient. As revealed by the comparison of DMS spectrum (Fig.2b) and $\mathrm{R}^{2}$ map, the location of zones is not directly deducible from the spectrum. The identification requires additional measurement data processing in addition to the visual investigation of spectrum.

In Fig. 4 there are presented fragment of positive ions DMS spectrum which are best in respect of linearity between the analyte concentration and the ionic current. Fragments include combinations of separation voltage and compensation voltage (SV,CV), where the liner fit was characterized by $\mathrm{R}^{2}>0.85$. This cut off threshold was chosen arbitrarily. Linearity zones in DMS spectrum were individually distinguished for methyl acetate, ethyl acetate, propyl acetate and butyl acetate.

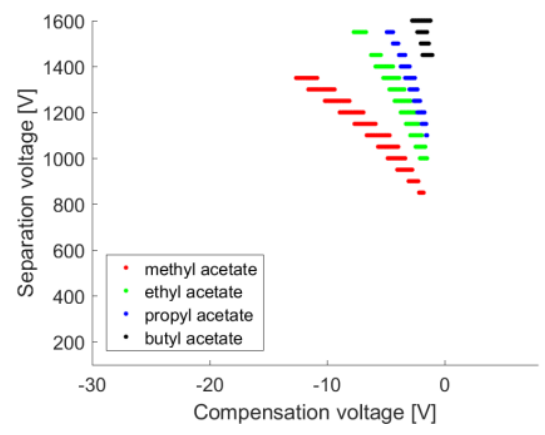

Fig. 4. Selection of best separation and compensation voltage combinations $(\mathrm{SV}, \mathrm{CV})$ for acetates. The selection was based on the criterion of $\mathrm{R}^{2}>0.85$. $\mathrm{R}^{2}$ indicates the quality of linear fit between analyte concentration and ionic current measured on the positive ions collecting electrode for the particular combination (SV,CV).

As presented in Fig. 4, the linearity of DMS response and analyte concentration was observed at different combinations (SV, CV) for different acetates. Based on the comparison of DMS spectrum fragments distinguished for methyl, ethyl, propyl and butyl acetate, it could be noted that for acetate with methyl group, linearity was achieved at relatively small separation voltages and relatively high compensation voltages. With the increase of the size of functional group in acetate molecule, from methyl to butyl group, linearity zones moved towards higher separation voltages and lower compensation voltages. In particular, the results obtained for butyl acetate suggest that more combinations (SV, $\mathrm{CV}$ ) would be available for the compound determination when extending the separation 
voltage range. The shift of peaks towards lower absolute values of compensation voltage indicates decreasing difference of ion mobility between low-field and high-field.

In Fig. 5.a there is presented the quality measure for linear fits, coefficient of determination $\left(\mathrm{R}^{2}\right)^{\prime}$ and in Fig. 4.b we show the analyte concentration prediction error (RMSE), which is made while applying linear models. Box plots were used to display the spread of these measures for models associated with best (SV,CV) combinations, as shown Fig. 4. The performance measures were compared for methyl, ethyl, propyl and butyl acetate.
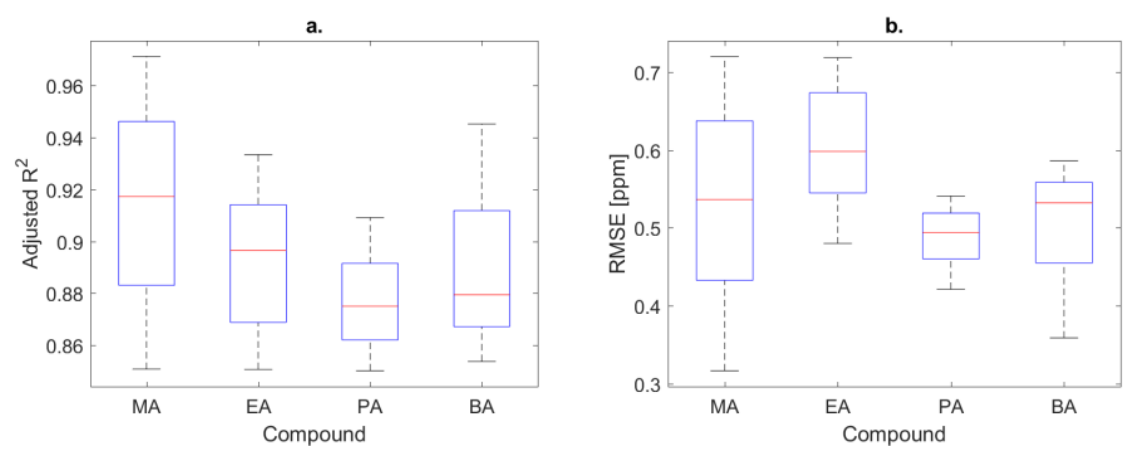

Fig. 5. a. Quality measure of the fit $\left(\mathrm{R}^{2}\right)$ for the pool of liner models associated with best combinations (SV,CV), see Fig. 4. b. Error of analyte concentration prediction using models associated with best combinations (SV,CV), see Fig. 4. Names of analytes were abbreviated: MAmethyl acetate, EA- ethyl acetate, PA- propyl acetate and BA- methyl acetate.

As shown in Fig. 5a, the response of DMS to acetates was highly linear, if the appropriate part of spectrum was exploited. Best linearity was achieved for methyl acetate. In this case, the average $\mathrm{R}^{2}$ for the group of best combinations $(\mathrm{SV}, \mathrm{CV})$ was $\mathrm{R}^{2}=0.92$. Second best results were attained for ethyl acetate, average $\mathrm{R}^{2}=0.90$. Butyl acetate was third $\left(\mathrm{R}^{2}=0.88\right)$ and for propyl acetate the linearity was weakest $\left(\mathrm{R}^{2}=0.87\right)$. When using these linear models acetates concentration prediction errors were in a range from 0.3 to $0.7 \mathrm{ppm}$, (see Fig. 5 b.).
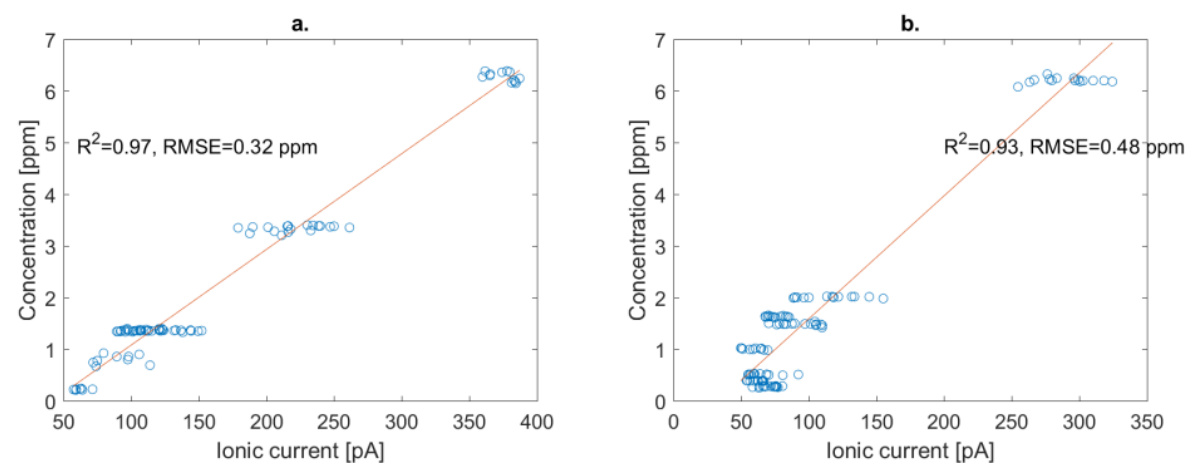

Fig. 6 Best linear fit between analyte concentration and the ionic current on positive ions collecting electrode. The fit was obtained for the best combination of (SV,CV). a. methyl acetate $(-3.4297,950)$, b. ethyl acetate $(-2.5,1100)$.

In Fig. 6 there are displayed best linear fits achieved for two acetates, namely methyl and ethyl acetate. They illustrate the fact that high linearity was attained in spite of considerable spread of DMS response, while exposing spectrometer to an individual standard gas mixture in a continuous manner. It can be noticed that in case of ethyl acetate (Fig.6 b.) measurements done at low concentrations (less than $1 \mathrm{ppm}$ ) did not fully match the linear relationship. The same problem was observed in case of propyl acetate measurement and it 
has to be further examined. In case of methyl acetate (shown in Fig. 6a) as well as butyl acetate this kind of deviation was not observed.

\section{Conclusions}

VOCs are an important group of pollutants. In order to control their environmental impact there are needed novel methods of measurement. In this wok, differential ion mobility spectrometry was considered in respect of measuring acetates in air. These compounds are released from a variety of sources, in particular indoors.

The performed study demonstrated that DMS offers linear response to methyl, ethyl, propyl and butyl acetate in the concentration range from $0.3 \mathrm{ppm}$ to $7 \mathrm{ppm}$. Positive ions spectrum has to be utilised for this purpose.

Achieving linearity requires that the appropriate part of spectrum is used. We showed that fragments of DMS spectrum, where proportionality to analyte concentration is achieved, are compound-specific. Upon increase of the size of functional group in acetate molecule, the useful part of spectrum moves towards higher separation voltage and lower compensation voltage.

The best linear dependencies were featured by the following coefficient of determination and root mean square errors of analyte concentration prediction: methyl acetate $\left(\mathrm{R}^{2}=0.97\right.$, $\left.\mathrm{RMSE}=0.32 \mathrm{ppm}\right)$, ethyl acetate $\left(\mathrm{R}^{2}=0.93\right.$, $\left.\mathrm{RMSE}=0.48 \mathrm{ppm}\right)$, propyl acetate $\left(\mathrm{R}^{2}=0.91, \mathrm{RMSE}=0.42 \mathrm{ppm}\right)$, butyl acetate $\left(\mathrm{R}^{2}=0.95, \mathrm{RMSE}=0.36 \mathrm{ppm}\right)$.

\section{References}

1. Y. Pang, M. Fuentes, P. Rieger, Atmos. Environ 122, 686-695 (2015)

2. S.J. Maisey, S.M. Saunders, N. West, P.J. Franklin, Atmos. Environ. 81, 546-553 (2013).

3. S.Chatani, S.N. Matsunaga, S. Nakatsuka, Atmos. Environ. 120, 38-50 (2015).

4. M. Kheirmand, A. Barkhordari, M.H. Mosaddegh, Z. Farajzadegan, Int. J Env. Health Eng. 3, 27 (2014).

5. Chien-Hou Wu, Chien-Tai Feng, Yu-Shiu Lo, Tsai-Yin Lin, Jiunn-Guang Lo, Chemosphere, 56, 71-80 (2004).

6. A. Szczurek, M. Maciejewska, Gas Sensor array with broad applicability, in Sensor Array,(InTech, 2012)

7. G.A. Eiceman, Z. Karpas, H.H. Hill Jr, Ion Mobility Spectrometry, 3rd edition (CRC Press/Taylor \& Francis Group, Boca Raton, 2014).

8. A.A. Shvartsburg, Differential ion mobility spectrometry: Nonlinear ion transport and fundamentals of FAIMS, (CRC Press, Boca Raton, 2009)

9. E.V. Krylov, E.G. Nazarov, R.A. Miller, Int. J. Mass Spectrom. 226, 76-85 (2007).

10. B.B. Schneider, E.G. Nazarov, F. Londry, P. Vouros, T.R. Covey, Mass Spectrom Rev. 35(6), 687-737 (2016).

11. J.L. Willems, M.M. Khamis, W.M. Saeid, R.W. Purves, G. Katselis, N.H. Low, A. ElAneed, Anal. Chim Acta 933 164-174 (2016).

12. A.Szczurek, M. Maziejuk, M. Maciejewska, T. Pietrucha, T. Sikora, Sens. Actuators B 152, 137-146 (2016)

13. M. Maziejuk J. Puton M. Szyposzyńska, Z. Witkiewicz, Talanta 144, 1201-1206, (2015). 